OPEN ACCESS

Edited by:

Rabia Miray Kisla Ekinci,

Ministry of Health, Turkey

Reviewed by:

Mehmet Yildiz,

Istanbul University Cerrahpasa, Turkey Gülen Gül Mert,

Consultant, Adana, Turkey

${ }^{*}$ Correspondence:

Meiping Lu

meiping/u@zju.edu.cn

${ }^{\dagger}$ These authors have contributed equally to this work

Specialty section:

This article was submitted to

Pediatric Rheumatology,

a section of the journal

Frontiers in Pediatrics

Received: 30 August 2021 Accepted: 17 November 2021 Published: 14 December 2021

Citation:

Shi N, Wang X, Zou L, Yang X, Ma Q and Lu M (2021) Case Report: Macrophage Activation Syndrome and

Widespread Neuroimaging Abnormality in Childhood-Onset Systemic Lupus Erythematosus. Front. Pediatr. 9:767115 doi: 10.3389/fped.2021.767115

\section{Case Report: Macrophage Activation Syndrome and Widespread Neuroimaging Abnormality in Childhood-Onset Systemic Lupus Erythematosus}

\author{
Nana Shi ${ }^{1+}$, Xiangying Wang ${ }^{2 \dagger}$, Lixia Zou ${ }^{3}$, Xinghui Yang ${ }^{4}$, Qian $\mathrm{Ma}^{3}$ and Meiping $\mathrm{Lu}^{3 *}$ \\ ' Department of Hematology-Oncology, Children' Hospital, Zhejiang University School of Medicine, National Clinical Research \\ Center for Child Health, Hangzhou, China, ${ }^{2}$ Hangzhou Children' Hospital, Hangzhou, China, ${ }^{3}$ Department of Rheumatology \\ Immunology and Allergy, Children' Hospital, Zhejiang University School of Medicine, National Clinical Research Center for \\ Child Health, Hangzhou, China, ${ }^{4}$ Department of Radiology, Children' Hospital, Zhejiang University School of Medicine, \\ National Clinical Research Center for Child Health, Hangzhou, China
}

Macrophage activation syndrome (MAS) and widespread brain lesions are rare and severe complications of childhood-onset systemic lupus erythematosus (SLE). We report an 11-year-old girl who presented with recurrent rashes for half a year and fever for 2 weeks. Clinical and laboratory features at admission pointed to the diagnosis of SLE and SLE-associated MAS. Cerebral magnetic resonance imaging taken on day 4 after admission showed abnormal signals. Glucocorticoid therapy was started on day 5. Two days later, the patient appeared weak and ill, then the next day she exhibited dizziness, drowsiness, apathia, and dysarthria. High-dose methylprednisolone, cyclophosphamide, and intravenous immunoglobulin were used to treat the patient, and intrathecal dexamethasone was given. The patient was discharged on day 30 after admission and showed complete clinical resolution and improved magnetic resonance imaging resolution.

\section{Keywords: macrophage activation syndrome, systemic lupus erythematosus, central nervous system,} neuroimaging, pediatric

\section{INTRODUCTION}

Systemic lupus erythematosus (SLE) is a clinically heterogeneous disease with variant manifestations and diagnosed on the basis of clinical and laboratory features. Macrophage activation syndrome (MAS) is a severe complication of rheumatic diseases and is currently classified among secondary forms of hemophagocytic lymphohistiocytosis (HLH). MAS manifests with fever, hepatosplenomegaly, central nervous system (CNS) dysfunction, hyperferritinemia pancytopenia, hepatic dysfunction, and coagulation abnormality. Compared with systemic juvenile idiopathic arthritis patients, SLE patients have significantly lower incidence of MAS, ranging from 0.9 to $9 \%(1-4)$. Widespread neuroimaging abnormality, which is a severe organ damage, was rarely reported in SLE-associated MAS. The present article reports a child with MAS and widespread neuroimaging abnormality as initial presentations at SLE diagnosis and describes the clinical course and management. 

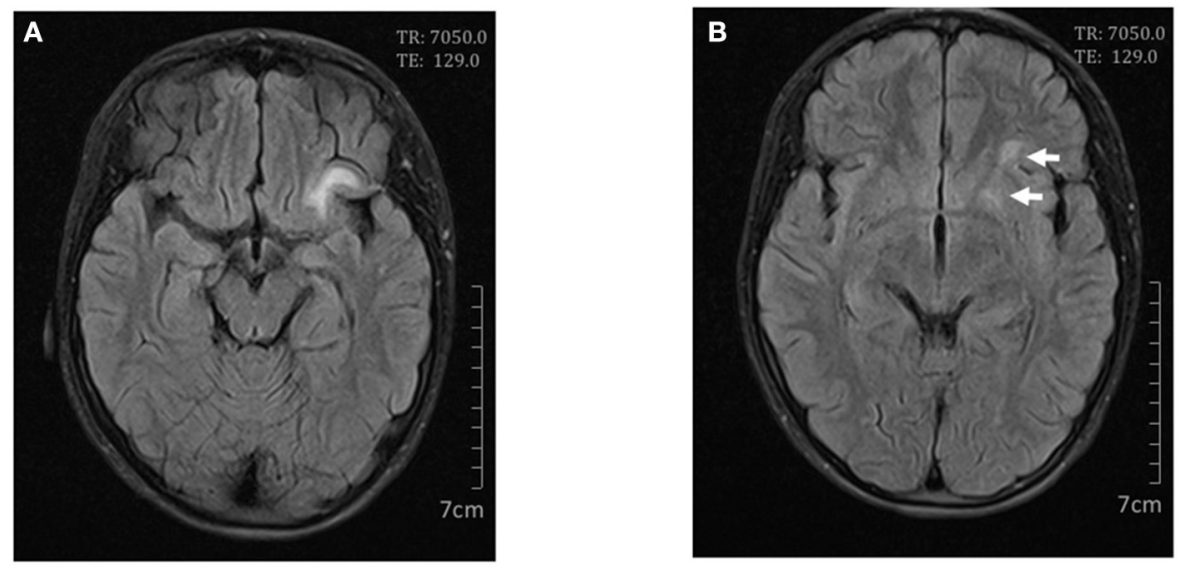

FIGURE 1 | Cerebral magnetic resonance imaging on day 4. Axial FLAIR scan showed high signals in the left frontal lobe (A) and lentiform nucleus (B).
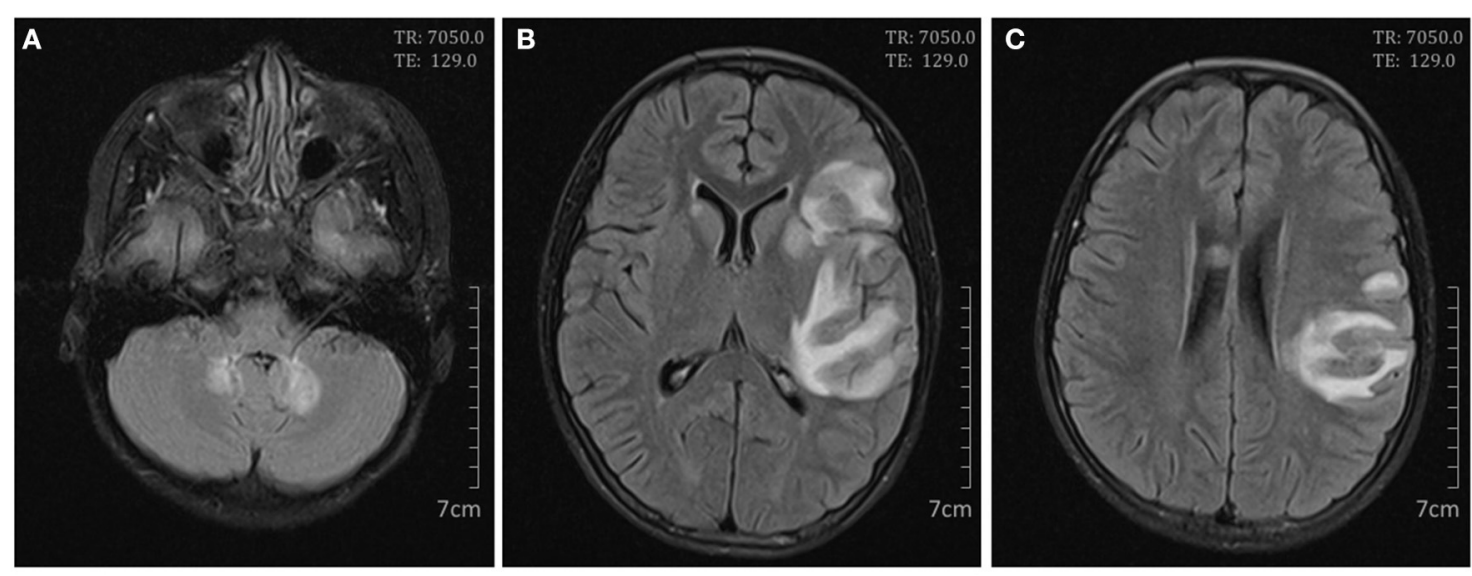

FIGURE 2 | Cerebral magnetic resonance imaging on day 9. Axial FLAIR scan showed high signals in the bilateral cerebellum (A); the bilateral basal ganglias and left frontal, temporal, insular, and parietal lobes (B); and the right corpus callosum and left parietal lobe (C).

\section{CASE DESCRIPTION}

An 11-year-old girl was admitted to our hospital because of recurrent rashes for half a year and high fever for 2 weeks. The patient often complained of knee and instep pain in the past few years, and she had acute pancreatitis twice in the past year. She had no history or family history of autoimmune disease, cancer, or tuberculosis.

The patient presented with fever, an ulcer on the lip, alopecia, malar rash, and rashes on the limbs. The initial laboratory results revealed leukopenia (white blood cell count $2.02 \times$ $10^{9} / \mathrm{L}$, lymphocyte count $0.70 \times 10^{9} / \mathrm{L}$ ), autoimmune hemolysis (hemoglobin $79 \mathrm{~g} / \mathrm{L}$ ), low C3 [0.26 g/L (normal range 0.9-1.8 $\mathrm{g} / \mathrm{L})]$, low C4 [0.04 g/L (normal range 0.1-0.4 g/L)], and

Abbreviations: CNS, central nervous system; FLAIR, fluid-attenuated inversion recovery; HLH, hemophagocytic lymphohistiocytosis; MAS, macrophage activation syndrome; MRI, magnetic resonance imaging; SLE, systemic lupus erythematosus. antibody abnormalities. The antinuclear antibody was positive with a titer of 1:160; the anti-double-stranded DNA and antiSmith antibodies were positive. Besides, laboratory data on admission also showed hyperferritinemia (ferritin $>1,500 \mu \mathrm{g} / \mathrm{L}$ ), hypertriglyceridemia $(4.87 \mathrm{mmol} / \mathrm{L})$, and elevated levels of aspartate aminotransferase $(123 \mathrm{U} / \mathrm{L})$ and lactate dehydrogenase $(621 \mathrm{U} / \mathrm{L})$. The fibrinogen level $(2.75 \mathrm{~g} / \mathrm{L})$ and urinalysis were normal. Cerebrospinal fluid and bone marrow examination on day 2 showed no infection or macrophages phagocytosing hematopoietic cells. Cerebral magnetic resonance imaging (MRI) was taken on day 4, revealing T2-white matte hyperintensities in her left frontal lobe and left lentiform nucleus (Figure 1). The presence of clinical and laboratory criteria led to the diagnosis of SLE and SLE-associated MAS.

The patient suffered from high fever twice a day after admission. Glucocorticoid therapy was started on day 5, with intravenous methylprednisolone $2 \mathrm{mg} / \mathrm{kg} / \mathrm{day}$. The next day her temperature returned to normal. 
On day 7 after admission the patient appeared weak and ill, then the next day she exhibited dizziness, drowsiness, apathia, and dysarthria, and her temperature reached $37.9^{\circ} \mathrm{C}$. The patient was given high-dose methylprednisolone pulse therapy (30 mg/kg/day) for 3 days. On day 9 the second cerebral MRI showed rapid progress (Figure 2), with multi-focal abnormal signals mainly involving white matter (bilateral temporal, cerebellum, insular lobes, and basal ganglias; left frontal and parietal lobes; right corpus callosum). The signals were hyperintense on fluid-attenuated inversion recovery (FLAIR). Cyclophosphamide $(8.5 \mathrm{mg} / \mathrm{kg} /$ day for 2 days) and intravenous immunoglobulin $(2 \mathrm{~g} / \mathrm{kg})$ were initiated in the therapy. On day 10 the patient showed significant improvements in all clinical conditions, including the rashes. On day 13 the third MRI demonstrated small infarcts in the left lentiform nucleus, left frontal lobe, temporal lobe, insular lobe, parietal lobe, and right corpus callosum, revealed by decrease in apparent diffusion coefficient in the gray matter, and an increased swelling in the surrounding white matter (Figure 3). She was given intrathecal dexamethasone once a week for three times. The second pulse of high-dose methylprednisolone was administered 1 week after the first pulse. Cyclophosphamide and immunoglobulin were administered with an interval of 2 weeks. On day 25 she had a fourth MRI scan and achieved neuroimaging improvements (Figure 4). The patient was discharged on day 30, with prednisone and addition of hydroxychloroquine.

\section{DISCUSSION}

The actual incidence of MAS in SLE patients is still unknown because of its under-recognition; the prevalence was reported ranging from 0.9 to $9 \%$. The mortality rate of MAS was high in patients with and without treatment (3). Early diagnosis of the MAS complication is not easy since there is no uniform diagnostic criteria, and on the other side, its similar laboratory and clinical features with the disease itself make the diagnosis more difficult (5). The HLH-2004 criteria are widely used in the diagnosis for MAS, but the sensitivity is not satisfactory; patients who meet the criteria usually have a more severe condition. In 2009, Parodi et al. set up diagnostic guidelines for MAS as complication for juvenile SLE (6). Compared with the HLH-2004 criteria, the guidelines included more variables, such as CNS dysfunction, aspartate aminotransferase, lactate dehydrogenase, and a higher sensitivity. Our patient met one clinical criterion and five laboratory criteria according to the 2009 guidelines and was diagnosed with MAS secondary to SLE (fever, cytopenia, increased aspartate aminotransferase, increased lactate dehydrogenase, hypertriglyceridemia, and hyperferritinemia).

We performed bone marrow puncture on day 2 after admission, demonstrating no hemophagocytosis. The fibrinogen level was normal on admission, but subsequent tests showed a gradual decline in fibrinogen level, which was $1.25 \mathrm{~g} / \mathrm{L}$ on day 12. The absence of hemophagocytosis and lack of fibrinogen consumption are unusual in fulminant MAS. However, the absence of hemophagocytosis on biopsy does not
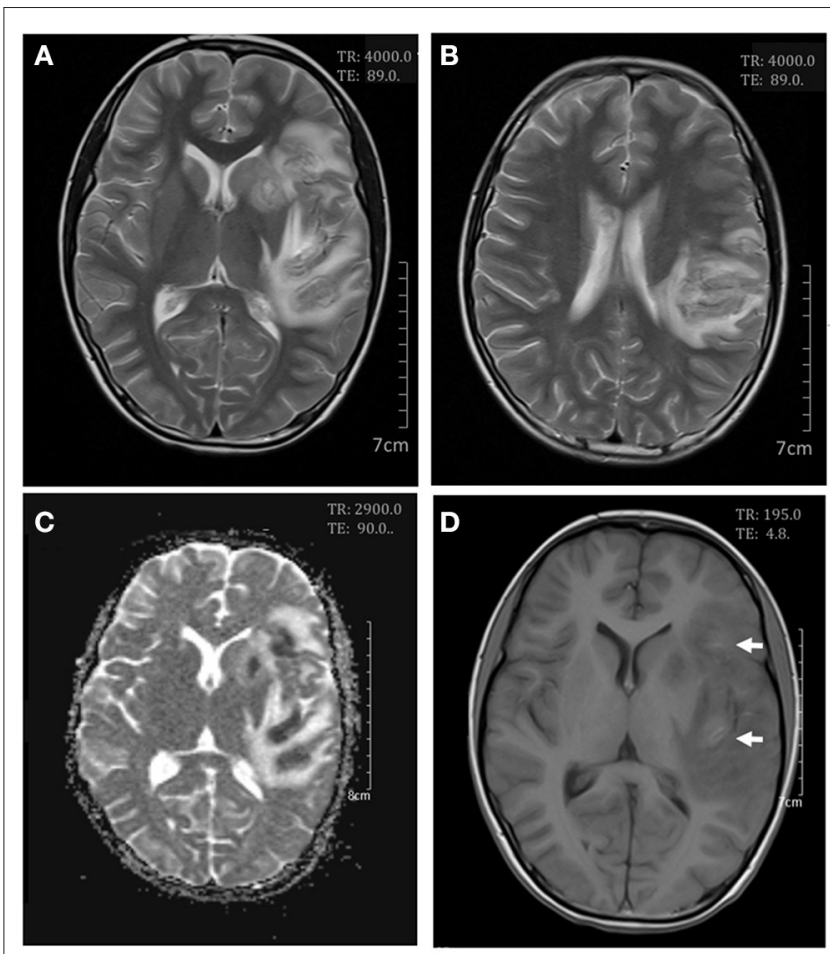

FIGURE 3 | Cerebral magnetic resonance imaging on day 13. Axial T2-weighted scan showed an increase in white matter swelling (A,B). Apparent diffusion coefficient maps demonstrated infarcts in the left lentiform nucleus and the frontal, temporal, insular, and parietal lobes (C). Axial T1-weighted scan showed small areas of hemorrhages in the frontal and insular lobes (D).

exclude the diagnosis of MAS. Research has established that hemophagocytosis often occurs in the later stage of the disease and is only present in $32-60 \%$ of patients $(3,7)$. Awaiting the change on bone marrow or organ biopsy could fatally delay diagnosis and treatment. Besides, Our patient had persistent fever and a sharp rise in ferritin level, which were the best parameters to discriminate MAS from active SLE with an almost $100 \%$ sensitivity and specificity (8), and subsequent results of fibrinogen consumption supported the diagnosis of MAS. Parodi et al. pointed out that the criteria may not be powerful enough to distinguish MAS from infectious complications. We found no evidence of infection in our patient, which further points to our diagnosis of MAS.

Our patient had CNS involvement, which was found both in SLE-associated MAS and active disease with neuropsychiatric lupus (NPSLE) as its clinical spectrum. Therefore, it is difficult to distinguish between these two conditions, which is quite different in patients with systemic juvenile idiopathic arthritis, in which a CNS dysfunction is not a manifestation of the active disease. The incidence of NPSLE events has been reported ranging from 14 to $67 \%$ in SLE patients (9-12), but only $13-38 \%$ of those events could be attributable to SLE (13); the main reason is that there are no clear standards to define. To improve the attribution, some models have been proposed $(14,15)$ but have not been widely used in clinical practice. The prevalence of 

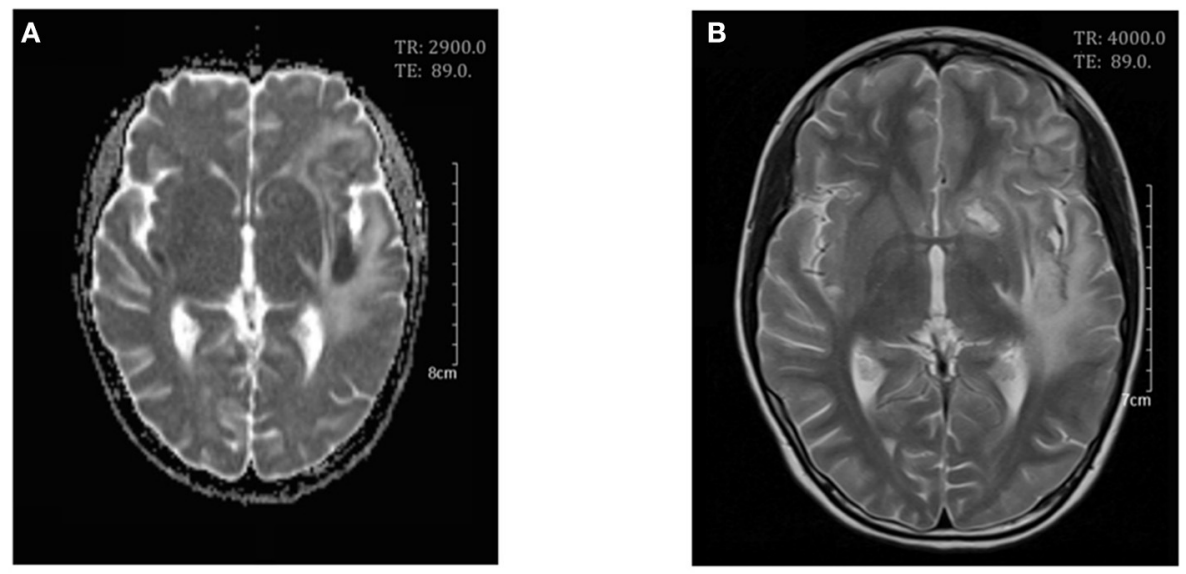

FIGURE 4 | Cerebral magnetic resonance imaging on day 25. Axial T2-weighed scan (A) and apparent diffusion coefficient (ADC) maps (B) showed a decrease in white matter swelling.

CNS involvement is $18-50 \%$ in SLE-associated MAS patients $(3,16)$; the attribution is also difficult because of its low incidence, and on the other side there is no specific biomarker or specific neuroimaging pattern. More than half of children with NPSLE have no conventional MRI abnormalities (17). The most common abnormalities are white matter hyperintensities, followed by brain atrophy, gray matter lesions, and basilar artery territory infarction (18). However, the neuroimaging patterns have rarely been studied in MAS patients, probably due to its low prevalence and the difficulty to distinguish it from NPSLE. In HLH patients, the most frequent changes are white matter T2 hyperintensity and encephalatrophy (19). Therefore, more research is needed in the future to meet the clinical need. In our case, we defined the CNS involvement secondary to MAS because it occurred at the time soon after the presentation of MAS and on the opinion of experts comprising two pediatric rheumatologists and one neurologist. Moreover, though there is no reliable definition, most experts of HLH agree that an abnormal MRI of the brain defines CNSHLH (20).

The neuroimaging of our patient showed widespread lesions, involving multi-lobes, which was rarely reported in MAS patients. We found three cases of MAS reporting CNS imaging but no case of SLE-associated MAS in the literature (21-23). Our patient presented multiple T2-hyperintense area mainly in the white matter, as well as small infarcts and microbleedings in the gray matter. The CNS abnormalities result from activated lymphocytes and macrophages infiltration in leptomeninges and brain parenchyma with multifocal necrosis and reactive astrogliosis. The patient had persistent high fever after admission, and a cerebral MRI was taken on day 4, revealing abnormal signals. Given the first MRI result, we were alert to severe disease progression when she developed CNS symptoms. Patients of MAS with CNS involvements were associated with a higher mortality rate (9). High dose of methylprednisolone, cyclophosphamide, and immunoglobulin were initiated. A rapid and complete recovery of CNS manifestations was achieved in the patient, proving our prompt recognition and management.

The present case reports a child of SLE with MAS and multifocal lesions on cerebral MRI as the onset presentations and suggests that we should have a great awareness of SLE-associated MAS. Early cerebral MRI examination of disease evaluation may be recommended in SLE-associated MAS patients for early recognition and treatment of severe cases.

\section{DATA AVAILABILITY STATEMENT}

The original contributions presented in the study are included in the article/supplementary material, further inquiries can be directed to the corresponding author.

\section{ETHICS STATEMENT}

The studies involving human participants were reviewed and approved by the Ethics Committee of the Children' Hospital, Zhejiang University School of Medicine (2020IRB-025). Written informed consent to participate in this study was provided by the participants' legal guardian/next of kin.

\section{AUTHOR CONTRIBUTIONS}

ML designed the work and revised the manuscript. NS and XW carried out the study and prepared the manuscript. XY performed and analyzed the magnetic resonance imaging. LZ and QM provided the clinical data. All authors contributed to the article and approved the submitted version.

\section{FUNDING}

The authors received financial support from Zhejiang Basic Public Welfare Research Project (LGF19H100002). 


\section{REFERENCES}

1. Minoia F, Davi S, Horne A, Demirkaya E, Bovis F, Li C, et al. Clinical features, treatment, and outcome of macrophage activation syndrome complicating systemic juvenile idiopathic arthritis: a multinational, multicenter study of 362 patients. Arthritis Rheumatol. (2014) 66:3160-9. doi: 10.1002/art.38802

2. Crayne CB, Albeituni S, Nichols KE, Cron RQ. The immunology of macrophage activation syndrome. Front Immunol. (2019) 10:119. doi: 10.3389/fimmu.2019.00119

3. Borgia RE, Gerstein M, Levy DM, Silverman ED, Hiraki LT. Features, treatment, and outcomes of macrophage activation syndrome in childhoodonset systemic lupus erythematosus. Arthritis Rheumatol. (2018) 70:61624. doi: 10.1002/art.40417

4. Fukaya S, Yasuda S, Hashimoto T, Oku K, Kataoka H, Horita T, et al. Clinical features of haemophagocytic syndrome in patients with systemic autoimmune diseases: analysis of 30 cases. Rheumatology (Oxford). (2008) 47:1686-91. doi: 10.1093/rheumatology/ken342

5. Lerkvaleekul B, Vilaiyuk S. Macrophage activation syndrome: early diagnosis is key. Open Access Rheumatol. (2018) 10:11728. doi: 10.2147/OARRR.S151013

6. Parodi A, Davi S, Pringe AB, Pistorio A, Ruperto N, Magni-Manzoni S, et al. Macrophage activation syndrome in juvenile systemic lupus erythematosus: a multinational multicenter study of thirty-eight patients. Arthritis Rheum. (2009) 60:3388-99. doi: 10.1002/art.24883

7. Cron RQ, Davi S, Minoia F, Ravelli A. Clinical features and correct diagnosis of macrophage activation syndrome. Expert Rev Clin Immunol. (2015) 11:104353. doi: 10.1586/1744666X.2015.1058159

8. Aytac S, Batu ED, Unal S, Bilginer Y, Cetin M, Tuncer $M$, et al. Macrophage activation syndrome in children with systemic juvenile idiopathic arthritis and systemic lupus erythematosus. Rheumatol Int. (2016) 36:1421-9. doi: 10.1007/s00296-016-3545-9

9. Bennett TD, Fluchel M, Hersh AO, Hayward KN, Hersh AL, Brogan TV, et al. Macrophage activation syndrome in children with systemic lupus erythematosus and children with juvenile idiopathic arthritis. Arthritis Rheum. (2012) 64:4135-42. doi: 10.1002/art.34661

10. Stéphan J, Koné-Paut I, Galambrun C, Mouy R, Bader-Meunier B, Prieur A. Reactive haemophagocytic syndrome in children with inflammatory disorders. A retrospective study of 24 patients. Rheumatology (Oxford). (2001) 40:1285-92. doi: 10.1093/rheumatology/40.11.1285

11. Gupta A, Tyrrell P, Valani R, Benseler S, Abdelhaleem M, Weitzman S. Experience with hemophagocytic lymphohistiocytosis/macrophage activation syndrome at a single institution. Pediatr Hematol Oncol. (2009) 31:814. doi: 10.1097/MPH.0b013e3181923cb4

12. Ravelli A, Magni-Manzoni S, Pistorio A, Besana C, Foti T, Ruperto N, et al. Preliminary diagnostic guidelines for macrophage activation syndrome complicating systemic juvenile idiopathic arthritis. J Pediatr. (2005) 146:598604. doi: 10.1016/j.jpeds.2004.12.016

13. Tay SH, Mak A. Diagnosing and attributing neuropsychiatric events to systemic lupus erythematosus: time to untie the Gordian knot? Rheumatology (Oxford). (2017) 56:i14-23. doi: 10.1093/rheumatology/k ex018

14. Bortoluzzi A, Scire CA, Bombardieri S, Caniatti L, Conti F, De Vita S, et al. Development and validation of a new algorithm for attribution of neuropsychiatric events in systemic lupus erythematosus. Rheumatology (Oxford). (2015) 54:891-8. doi: 10.1093/rheumatology/k eu384
15. Hanly JG, Urowitz MB, Gordon C, Bae SC, Romero-Diaz J, SanchezGuerrero J, et al. Neuropsychiatric events in systemic lupus erythematosus: a longitudinal analysis of outcomes in an international inception cohort using a multistate model approach. Ann Rheum Dis. (2020) 79:35662. doi: 10.1136/annrheumdis-2019-216150

16. Gormezano NW, Otsuzi CI, Barros DL, da Silva MA, Pereira RM, Campos LM, et al. Macrophage activation syndrome: a severe and frequent manifestation of acute pancreatitis in 362 childhood-onset compared to 1830 adult-onset systemic lupus erythematosus patients. Semin Arthritis Rheum. (2016) 45:706-10. doi: 10.1016/j.semarthrit.2015.10.015

17. Al-Obaidi M, Saunders D, Brown S, Ramsden L, Martin N, Moraitis E et al. Evaluation of magnetic resonance imaging abnormalities in juvenile onset neuropsychiatric systemic lupus erythematosus. Clin Rheumatol. (2016) 35:2449-56. doi: 10.1007/s10067-016-3376-9

18. Postal M, Tamires Lapa A, Reis F, Rittner L, Appenzeller S. Magnetic resonance imaging in neuropsychiatric systemic lupus erythematosus: current state of the art and novel approaches. Lupus. (2017) 26:51721. doi: 10.1177/0961203317691373

19. Ma W, Li XJ, Li W, Xiao L, Ji XJ, Xu Y. MRI findings of central nervous system involvement in children with haemophagocytic lymphohistiocytosis: correlation with clinical biochemical tests. Clin Radiol. (2021) 76:159 e159e117. doi: 10.1016/j.crad.2020.09.009

20. Horne A, Wickstrom R, Jordan MB, Yeh EA, Naqvi A, Henter JI, et al. How to treat involvement of the central nervous system in hemophagocytic lymphohistiocytosis? Curr Treat Options Neurol. (2017) 19:3. doi: 10.1007/s11940-017-0439-4

21. Gianella S, Schaer DJ, Schwarz U, Kurrer M, Heppner FL, Fehr J, et al. Retinal microangiopathy and rapidly fatal cerebral edema in a patient with adultonset Still's disease and concurrent macrophage activation syndrome. Am J Hematol. (2008) 83:424-7. doi: 10.1002/ajh.21084

22. El-Bitar MK, Muwakkit SA, Hourani R, Abboud MR, Saab R, Dabbagh O. Severe cerebral vaso-occlusive disease in macrophage activation syndrome. Pediatr Neurol. (2010) 42:283-6. doi: 10.1016/j.pediatrneurol.2009.11.007

23. Lilleby V, Haydon J, Sanner H, Krossness BK, Ringstad G, Flato B. Severe macrophage activation syndrome and central nervous system involvement in juvenile dermatomyositis. Scand J Rheumatol. (2014) 43:1713. doi: $10.3109 / 03009742.2013 .863968$

Conflict of Interest: The authors declare that the research was conducted in the absence of any commercial or financial relationships that could be construed as a potential conflict of interest.

Publisher's Note: All claims expressed in this article are solely those of the authors and do not necessarily represent those of their affiliated organizations, or those of the publisher, the editors and the reviewers. Any product that may be evaluated in this article, or claim that may be made by its manufacturer, is not guaranteed or endorsed by the publisher.

Copyright (C) 2021 Shi, Wang, Zou, Yang, Ma and Lu. This is an open-access article distributed under the terms of the Creative Commons Attribution License (CC BY). The use, distribution or reproduction in other forums is permitted, provided the original author(s) and the copyright owner(s) are credited and that the original publication in this journal is cited, in accordance with accepted academic practice. No use, distribution or reproduction is permitted which does not comply with these terms. 